http://dx.doi.org/10.5902/2236117012435

Revista do Centro do Ciências Naturais e Exatas - UFSM, Santa Maria

Revista Eletrônica em Gestão, Educação e Tecnologia Ambiental - REGET

e-ISSN 22361170 - V. 18 n. 1 Abr 2014, p.381-387

\title{
Construção de política para gestão de resíduos na Universidade de São Paulo como modelo para implementação da PNRS em IES
}

\author{
Construction of a Residue Management Policy in the University of São Paulo: A model for the implementation of the NSRP in HEI \\ Patrícia G. Moreira', Patricia B. Di Vitta', Adriano C. Pimenta', Ana Maria de Meira', Arthur R. Silva', \\ Cecília H. T. Cavalheiro', Clara M. Zorigian', Daniela C. Sudan', Elizabeth T. Lima', \\ Glauco A. Tavares'; Miguel Cooper', Patrícia C. S. Leme', Simone B. J. Simonelli', \\ Vera Gandra C. Albuquerque', Welington B. C. Delitti' \\ ' Membros do Grupo de Trabalho de Resíduos da Superintendência da Gestão Ambiental, Universidade de São Paulo, São Paulo
}

\begin{abstract}
Resumo
O cenário atual da Universidade de São Paulo (USP) tem, isoladamente, diversas ações ambientais em diferentes frentes, de acordo com a realidade de seus campi. Em 2010, a USP designou um coordenador de Gestão Ambiental junto à Reitoria. No ano seguinte, regulamentou a Política Ambiental da USP, que visa promover a sustentabilidade ambiental nos campi. Em 2012, foi criado um órgão institucional responsável pela gestão ambiental, a Superintendência de Gestão Ambiental (SGA), que tem como premissa ditar normas para questões ambientais em consonância com a política ambiental proposta. Esta superintendência formou, em 2012, um Grupo de Trabalho para cuidar das questões relativas aos resíduos gerados na USP. Tal grupo, composto por especialistas na área, sob a coordenação de um assessor de gabinete da própria SGA, priorizou desenvolver, através de processos participativos, estratégias e procedimentos para elaboração e implantação de uma Política de Gestão de Resíduos (PGRUSP). Esta Política, aos moldes de um trabalho anterior (PUSP-C, 2010), tem como proposta adequar as especificidades contidas na Política Nacional de Resíduos Sólidos, para o contexto universitário.Uma versão inicial da PGRUSP foi apresentadapara a comunidade acadêmica em um Fórum promovido pela SGA. Nesta ocasião, representantes de todas as categorias advindos dos diversos campi da USP, além de outros da comunidade externa, fizeram considerações sobre o conteúdo desta versão, que foi, então, reformulada. A PGRUSP prevê a elaboração dos planos de gerenciamento de resíduos em todas as unidades pertencentes à USP. Neste momento, o Grupo de Trabalho está elaborando diretrizes para a capacitação da comunidade acadêmica para a realização de um diagnóstico de resíduos, com intuito também de mobilizar e conscientizar sobre os ditames da PGRUSP. A partir da capacitação e da elaboração dos planos de gerenciamento de resíduos, pressupõe-se um aprendizado sobre questões práticas da gestão de resíduos. A geração de dados e de indicadores fornecerá embasamento para uma implementação contínua e eficaz da política. Para sistematização destes dados, está sendo desenvolvida uma plataforma virtual unificada. Assim, pretende-se controlar tanto os insumos utilizados como a geração, o armazenamento, os tratamentos e os descartes relacionados aos resíduos gerados em nossas Unidades. Pretende-se, ainda, com esta sistematização, fornecer informações que permitam à SGA atomar medidas norteadoras para melhora na gestão dos resíduos nos campi, e,consequentemente, minimizar impactos ambientais causados em todos os níveis e atividades.
\end{abstract}

Palavras-chave: Instituição Ensino Superior (IES), Gestão de Resíduos, Gestão Ambiental

\begin{abstract}
The actual scenario of the University of São Paulo (USP) has, in an isolated form, various environmental initiatives in diverse fronts, according to the reality of its campi. In 2010, USP designated an Environmental Management coordinator attached to the university's rectory. The following year USP approved the its Environmental Policy, that promotes environmental sustainability in all its campi. In 2012 the Environmental Management Superintendence (SGA) was created as an office responsible for environmental management of the university, with the objective of developing norms for environmental issues in agreement to the proposed environmental policy. This superintendence created in 2012 a Working Group for dealing with issues related to solid residues generated in USP. This group, composed of specialists in this area and coordinated by an member of the SGA, developed, using participative processes, strategies and procedures for the elaboration and implementation of the Solid Residue Management Policy (PGRUSP). This policy following a previous model (PUSP-C, 2010), has as objective adequate the National Solid Residue Policy (PNRS) to the university's environment. An initial version of the PGRUSP was presented to the academic community in a Forum promoted by the SGA. In this occasion, representatives of all the categories coming from the university's different campi, apart from members of the external community, analyzed the document and made suggestions on its contents that were later included in the final document. PGRUSP determines the elaboration of Residue Management Plans in all the units that belong to USP. At this moment, the Working Group is elaborating directives for the training of the academic community for the execution of an ample residue diagnosis, with the objective of also mobilizing and publicizing the directives of the PGRUSP. The training and residue management plan construction presupposes learning about the practical issues of residue management. Data production and indicator construction will be the basis for the continuous and efficient implementation of this policy. For the ordering of the data a virtual platform is being developed. With such a platform, USP pretends to control generation, stocking, treatment and destination of Solid Residues produced in its academic units. It pretends with this database, to generate information that will permit the SGA to make decisions that will improve residue management in USP's campi and, consequently, minimize environmental impacts caused in all levels of its activities.
\end{abstract}

Keywords: Higher Education Institution (HEI), Waste Management, Environmental Management 


\section{INTRODUÇÃO}

Segundo Tauchen e Brandli (2006) p. 505, as IES podem ser comparadas a pequenos núcleos urbanos que desenvolvem atividades de ensino, pesquisa, extensão além de outras correlacionadas à sua operação, como por exemplo alimentação, alojamento, financeiras. Além disso, as IES utilizam por práticas ambientais isoladas, tendo em vista que as leis, em geral, são instituídas após sua implementação e funcionamento. Tal situação leva algumas universidades a atenderem a legislação sem necessariamente se preocupar com a promoção de práticas preventivas.

Em geral, os resíduos gerados em ambiente universitário são caracterizados por uma matriz complexa e heterogênea devido à complexidade e particularidade de suas atividades e, portanto, torna-se um desafio para toda comunidade acadêmica promover o gerenciamento adequado em todos os setores e atividades. Por isso, as IES devem utilizar a gestão de seus resíduos como parte da gestão acadêmica para desenvolver e implementar políticas relacionadas aos aspectos e impactos resultantes de suas atividades. (Conto, 2010)

Sabe-se, no entanto, que não há legislação específica definida para as peculiaridades dos resíduos gerados em IES e nem fiscalização adequada. Portanto, uma das formas das IES demonstrarem seu comprometimento com as questões ambientais é através da adoção de políticas institucionais e elaboração de um sistema de gestão ambiental que atenda às suas especificidades. Para promover essa ação com eficácia é importante o envolvimento dos diversos setores, sobretudo a Alta Administração/ Reitoria (Giloni-LIMA E LIMA, 2008; Silva e Mendes 2009).

Corrêa (2009) p. 20 enfatiza que a universidade tem como vantagem ser constituída por uma comunidade acadêmica composta das diversas áreas do conhecimento e que isto potencializa o processo de construção de políticas para gestão de resíduos. Além disso, segundo Tauchen e Brandli p. 01 (2006), a implantação de políticas de gestão de resíduos inicia um processo de conscientização em todos os níveis e atividades (administradores, professores, funcionários e alunos) contribuindo para uma melhoria na qualidade ambiental da instituição.

Aliado a isso Corrêa apud Corrêa (2012) p. 10 ressalta que a construção de uma política de gestão de resíduos nas IES com enfoque na educação ambiental, promove um envolvimento articulado no ambiente universitário. Desta forma permite o estabelecimento de uma consciência por parte dos atores envolvidos da sua responsabilidade enquanto gerador de resíduo no contexto de suas atividades nos diversos setores, desencadeando um processo educativo contínuo e efetivo que conduzirá à desacomodação.

Devido à falta de estudos sistematizados que examinem os processos produtores de resíduos em Universidades, as soluções na maioria das vezes são pensadas quando o resíduo é gerado e esse é um dos motivos que impedem a participação da comunidade na intervenção do problema, uma vez que ele já foi gerado. Além disso, não há clareza do papel dos diferentes agentes responsáveis pela geração de resíduos nas atividades de ensino, pesquisa e extensão aliado à falta de interlocução entre os mesmos (Conto, 2010).

\section{POLÍTICA DE GESTÃO DE RESÍDUOS DA USP}

\section{I HISTÓRICO}

A USP é composta pelos diversos campi em cidades distintas no Estado de São Paulo. Todas as Unidades, Museus, Órgãos de Integração, Órgãos Complementares, prestadores de serviços, cessionários e demais órgãos ou instituições que ocupam espaços físicos dentro da USP possuem vários programas ambientais com a finalidade de reduzir os impactos por ela causados em suas atividades. Portanto, o cenário atual da USP tem hoje, diversas ações ambientais importantes em diferentes frentes, de acordo com a realidade de cada campi.

Em 2009 a USP aprovou a Proposta de uma Política Ambiental. Em 2010, ano da publicação da PNRS foi criado um Grupo de Trabalho de Recursos Naturais e Resíduos da USP, o qual elaborou um documento intitulado "A USP e a especificidade de seus campi $2^{a}$ Edição Campus 
Sustentável" $1^{2}$ durante o Fórum Permanente Sobre Uso de Espaço Público da USP, na tentativa de conhecer as iniciativas e integrar experiências, vislumbrando a possibilidade da institucionalização da PNRS aos moldes da Universidade. Neste mesmo ano, com a Portaria do Reitor designou um coordenador de Gestão Ambiental junto à Reitoria e em 2011 regulamentou a criação do órgão institucional responsável pela gestão ambiental, a Superintendência de Gestão Ambiental da USP (SGA/ USP), a qual tem como premissa ditar normas para questões ambientais em consonância com a Política Ambiental, qual seja de promover a sustentabilidade ambiental nos campi da USP.

\subsection{Aspectos estratéGicos}

Em 2012 entrou em vigor a estrutura operacional da SGA/USP e a primeira fase do planejamento realizada pela SGA/USP para Gestão dos Resíduos consistiu na formação de um Grupo de Trabalho composto por especialistas na área dentro dos campi sob a coordenação de um professor titular designado como assessor de gabinete junto à superintendência de Gestão Ambiental. O grupo denominado "GT Resíduos da USP" priorizou desenvolver, através de processos participativos, estratégias e procedimentos para elaboração e implantação de uma Política de Gestão de Resíduos para Universidade. Esta Política tem como proposta adequar às especificidades contidas na PNRS para o contexto universitário.

Na segunda fase do planejamento, a participação da comunidade acadêmica se deu através de um Fórum $2^{3}$ promovido pela SGA. O evento contou com a presença de 110 representantes das diferentes categorias de todos os campi da USP, no qual todos os envolvidos diretos puderam participar da elaboração da versão preliminar deste documento que sofreu posteriormente alterações.

A versão preliminar da Política de Gestão de Resíduos da USP (PGR/USP) encaminhada às instâncias jurídicas da Universidade e será instituída através de uma Resolução publicada no Diário Oficial da União. A implantação da Política tem previsão de entrar em vigor no primeiro semestre de 2014 e será regulamentada através de Portarias publicadas pelo Reitor ou pelo Superintendente de Gestão Ambiental. Da mesma forma que a PGR-USP foi elaborada aos moldes da PNRS, as Portarias terão como base o Decreto 7.404/10.

A PGR/USP prevê a elaboração dos Planos de Gerenciamento de Resíduos em todas as unidades pertencentes à USP. Considerando como instrumento primordial para implantação da Política universitária, o grupo elaborou o documento preliminar da Portaria para Elaboração dos Planos de Gerenciamento de Resíduos da USP.

Com isso, a USP está implantando uma Política de Gestão de seus Resíduos, revendo práticas e condutas comumente adotadas por todos os setores da Instituição, com o intuito de organizar as fontes geradoras de resíduos e desenvolver procedimentos para o gerenciamento dos mesmos.

\section{ANÁLISE DO PROCESSO DE IMPLANTAÇÃO DA POLITICA DE GESTÃO DE RESÍDUOS DA USP}

O processo de implantação da PGR/USP demanda etapas para sua realização. Considerando como sendo a etapa inicial do processo analisado, serão feitos alguns apontamentos acerca dos limites e dificuldades encontrados para Implantação de uma Política de Gestão de Resíduos na USP a partir de uma visão sistêmica baseados na teoria e prática:

Resistência à mudanças de comportamento da comunidade acadêmica. Em geral devido aos hábitos e atitudes previamente estabelecidos e sistematizados que muitas vezes conduzem à comodidade somando-se também aos trâmites burocráticos (Spelleberg et al 2004 p. 127 \& Nicolades 2006 p.148);

Falta de consciência por parte da comunidade universitária no processo de construir, implantar e manter a política ambiental. Isso se dá pela dificuldade de inserção dos colaboradores nos processos participativos de forma pró-ativa e também pela limitação orçamentária para compor quadro de funcionários habilitados e estrutura logística para a implantação da política;

Estrutura descentralizada e fragmentada nas instituições. Esta situação estabelece uma dificul-

\footnotetext{
${ }^{2}$ Relatório interno não publicado.

${ }^{3}$ Workshop denominado "Iniciativas para Construção da Política de Gestão de Resíduos da USP " realizado 25 de abril de 2013, na sala do Conselho Universitário do Campus São Paulo, contando com a presença de cerca de 110 representantes de todos os campi da USP.
} 
dade de reconhecimento dos responsáveis e mudança de gestores ao longo do processo, onde os novos em geral resistem em sustentar ações que vinham sendo executadas, preferindo criar novas estratégias (Spelleberg et. al 2004 p. 127 \& Nicolades 2006 p.148);

Dificuldade de inserir a dimensão ambiental nos diferentes setores da instituição e nos projetos pedagógicos e de pesquisa refletindo numa resistência dos docentes em incorporar a questão ambiental em suas discussões e priorizar a alocação de verbas institucionais para investimentos nessa questão. Este ponto está diretamente ligado à necessidade de um programa de educação ambiental permanente em que as questões ambientais passam a fazer parte da logística de planejamento da instituição como um todo (Spelleberg et al 2004 p. 127 \& Nicolades 2006 p.148);

Desperdício ou manejo inadequado de verba recebida pelos órgãos de fomento e pesquisa. Em geral, quando utilizada para armazenamento de produtos, contribuindo para o aumento da geração e custo para instituição no momento da disposição final ambientalmente adequada;

Falta de incentivo pelos órgãos de fomento que priorizam a valorização de publicações associadas somente à área de atuação do profissional. Esta situação está associada ao desconhecimento da importância de estudos sobre a temática de Resíduos em Universidades. Esta temática multidisciplinar deveria ser parte integrante das atividades de pesquisa, podendo estar associada direta ou indiretamente aos projetos, uma vez que todos, de alguma forma, são geradores de resíduos. Sendo assim, a prioridade induzida pelos órgãos em questão torna-se contraditória, pois projetos sobre a temática em universidades podem trazer consigo dados que direcionem ao melhor aproveitamento da verba disponibilizada;

Ainda há uma falta de comprometimento da administração e comunidade universitária com ações e práticas que visam à sustentabilidade. Esta situação está diretamente ligada ao fato da temática não estar incorporada nas questões cotidianas, entendendo que se o indivíduo se apropriar do conhecimento poderá trazer ganhos comportamentais e inovadores em sua vida pessoal que refletirá em sua rotina ou vice-versa (Spelleberg et. al 2004 p. 127 \& Nicolades 2006 p.148);

Falta de tempo das pessoas envolvidas devido à demanda crescente em suas atividades que se fazem valer pela produtividade. A sobrecarga de atividades dos profissionais que se fazem valer pela produção intelectual na área acadêmica. Além de suas atividades, os docentes com cargas horárias de ensino e pesquisa assumem cargos administrativos pela estrutura da universidade que se intensifica, muitas vezes, com a limitação de novas contratações (Spelleberg et al 2004 p. 127 \& Nicolades 2006 p.148).

A partir das dificuldades encontradas, serão elencados os desafios e perspectivas que poderão contribuir na eficácia da Implantação da Política de Gestão de Resíduos na Universidade de São Paulo:

Definição clara das responsabilidades na gestão de resíduos da universidade (administração superior, professores alunos e funcionários) (Dias apud Conto, 2010 p.266);

Criação de verba orçamentária específica em todas as unidades e setores para o gerenciamento dos resíduos;

Implementação do diagnóstico da situação atual e inserção dessa rotina, a qual fornecerá indicadores como subsídios para cumprimento de metas e melhoria contínua durante a gestão dos resíduos; (BARBIERI apud CORREAA, 2009 p. 34)

As despesas com gerenciamento de resíduos tem custo elevado e devem, portanto, ser rateadas entre as unidades, além disso, todas as unidades devem saber para onde e como seus resíduos estão sendo enviados e tratados (Teixeira apud Conto 2010 p. 124);

Elaboração de um sistema de informações Unificado de Resíduos, que possa controlar os insumos utilizados, a entrada, estoque, descrição de tratamentos e descartes destes resíduos e cujos relatórios com dados gerais possam estar disponíveis para a comunidade USP;

Criação de um banco de dados sobre publicações relacionadas a programas e ações ambientais envolvidos na Universidade (CONTO, 2010 p. 28);

Incentivo ao desenvolvimento de teses, dissertações, monografias, estágios, trabalhos de conclusão de cursos e projetos de pesquisa com temas ambientais e em especial com a Universidade como objetivo de estudo (CONTO, 2010 p. 28);

Realização de ações de educação ambiental de forma contínua e permanente que tenha capacidade de mobilizar alunos e professores para o desenvolvimento de tecnologias apropriadas para o reaproveitamento de resíduos em suas atividades com valorização a utilização de técnicas como fórum de discussões (Dias apud Conto 2010 p.267); 
Estímulo para as ações que resultem na minimização de geração de resíduos, podendo-se pensar na possibilidade de criar um setor específico de pesquisa em tecnologias mais limpas adaptadas ao contexto universitário. Tal ação visa contribuir para diminuição do custo financeiro do tratamento e disposição dos resíduos para as unidades e, consequentemente, para a Universidade;

Apresentação da Política Ambiental e Política de Gestão de Resíduos da Universidade em disciplinas básicas dos cursos, contemplando a informação nos programas de ensino para todos os alunos, prevendo ainda a divulgação entre todos os professores e funcionários (CONTO, 2010 p.28);

Inclusão na grade curricular de disciplina que possibilitem o aluno da graduação ser conscientizado sobre a temática de "Sustentabilidade na Universidade" associando a questão dos "Resíduos em Universidades" (CONTO, 2010 p. 24);

Os projetos de pesquisa e os programas de ensino e extensão precisam ser repensados no sentido de serem desenvolvidos com o menor impacto ambiental, independentemente da área do conhecimento ao qual ele estiver vinculado. Todo projeto, em todo seu desenvolvimento, deve estabelecer um compromisso com o meio ambiente, demonstrando medidas de prevenção de geração de resíduos com técnicas utilizadas para o manejo correto dos mesmos;

Novas políticas institucionais como de compras, comunicação, pesquisa, recursos humanos, planejamento, infra-estrutura, administrativa devem estar relacionadas a gestão de resíduos (CORRÊA, 2009 p.191.);

Elaboração e inserção de um sistema de verificação que permita visualizar a eficiência na Gestão de Resíduos. Pensando no contexto da sustentabilidade que se expande também ao contexto social e econômico universitário, a discussão poderia se voltar, inclusive para uma proposta de elaboração de um relatório de sustentabilidade para universidade. O cenário atual das plataformas existentes para elaboração dos relatórios não está totalmente adaptada a realidade de uma IES, se fazendo valer ainda, de parâmetros industriais e mercadológicos. Associado a isso, outro desafio está na seleção de bons indicadores ambientais para a análise dos impactos ambientais na Universidade;

Incentivo por parte dos órgãos de fomento para projetos de pesquisa das áreas afins associados ao tema "Gestão de Resíduos em Universidades".

Levando-se em consideração que a análise do processo em questão se deu de forma parcial pois trata-se da fase inicial da Implantação da Política, pôde-se verificar a necessidade de uma proposta educativa que promova a participação de todos os envolvidos e que seja baseada na problematização das vivências dentro ambiente universitário.

Deste modo, segundo Conto, 2010, poderá proporcionar um questionamento coletivo de atitudes e comportamentos diante do contexto ambiental universitário valorizando o diálogo crítico, construtivo e transformador que poderá conduzir à desacomodação.

\section{CONSIDERAÇÕES FINAIS}

Apesar das dificuldades apontadas, a Universidade conta com um grande avanço em pouco tempo, pois iniciou o processo de institucionalização da PNRS e criação de um órgão ambiental responsável, centralizador e de apoio dentro da Universidade em uma única gestão.

As iniciativas até então, eram individualizadas de acordo com a realidade dos campi e suas unidades, porém, sem a centralização dessas informações e apoio de um órgão institucional. Hoje, essas iniciativas poderão ser aproveitadas e tidas como conhecimento de todos através de mecanismos de divulgação, encontros, capacitação, fóruns, dentre outras ações que, além de contemplar as soluções técnicas de um trabalho educativo, podem estimular os envolvidos a discutir e refletir, a partir de suas vivências nas atividades de ensino, pesquisa e extensão e prestação de serviço.

Assim como a PNRS trouxe um novo olhar da sociedade para com seus resíduos, a criação de uma Política de Gestão de Resíduos para Universidade pela SGA/USP, também poderá trazer para a Universidade o mesmo enfoque, a partir do momento que todos serão mobilizados para a elaboração do diagnóstico de resíduos e levantamento da situação atual. Com isso, serão criados mecanismos que possibilitem uma nova conduta no que se refere ao Gerenciamento de Resíduos nas Unidades e consequentemente maior comprometimento com as questões ambientais abordadas na Política Ambiental proposta. 
Portanto, a implementação do Gerenciamento de Resíduos em toda Universidade possibilitará conhecer o contexto da instituição, perceber fragilidades e possibilidades. Os resultados começarão a surtir efeito quando a comunidade universitária passar a incorporar a dimensão ambiental em suas políticas, planejamentos e atividades, problematizando a questão a partir de um diálogo articulado, entre toda comunidade universitária na busca de soluções para os problemas ambientais. (CORREAA, 2009).

Nesse sentido somente através de um esforço coletivo, unindo experiências e ações individuais e dos grupos, aliado ao órgão centralizador, será possível promover as necessárias mudanças de atitudes no âmbito universitário.

A partir deste trabalho, outros mecanismos de difusão do conhecimento sobre temática de Resíduos na IES podem ser introduzidos, como por exemplo através da criação de vídeos institucionais educativos, construção de programa de divulgação na rádio universitária USP, jornais, palestras e fóruns sobre a temática com intuito de promover a discussão sobre a temática da Sustentabilidade nas Universidades a partir da Gestão dos seus Resíduos.

\section{REFERÊNCIAS}

BRASIL; Lei 12.305, de 02 de agosto de 2010. Institui a Política Nacional de Resíduos Sólidos; altera a Lei n.9.605, de 12 de fevereiro de 1998; e dá outras providências. Diário oficial da União: Brasília. Disponível em: $<$ http://www.planalto.gov.br/ccivil 03/ ato2007-2010/2010/lei/112305.htm> Acesso em 12 Abr. 2013.

CONTO SM org. Gestão de resíduos em universidades: Uma complexa relação que estabelece entre heterogeneidade de resíduos, gestão acadêmica e mudanças comportamentais. In: Gestão de Resíduos em Universidades. p. 17-32. Ed. EDUCS. 2010.

Gestão de Resíduos em Universidades. Revista Rosa dos Ventos. v. 4 (I), p.110-113. Jan-Junh 2012. Disponível em: < http://ucs.br/etc/revistas/index.php/rosadosventos/article/viewFile/1453/pdf 68> Acesso em 14 Mai. 2013.

CorrÊa LB. Construção de políticas para a gestão dos resíduos em uma instituição de ensino superior na perspectiva da educação ambiental. Rio Grande: Universidade Federal do Rio Grande; 2009. Disponível em: < http://www.ufrgs.br/sga/SGA/materialdeapoio/textos/textosapoio/links/tese $\% 201$ uciara $\% 20 \mathrm{bilhalva \% 20}$ correa.pdf> Acesso em: 12 Julh 2013.

CORREAA LB; LUNARDI VL; JACOBI PR. Educação Ambiental na construção de políticas para gestão dos resíduos em uma instituição de ensino superior. Revista Brasileira de Educação Ambiental, Rio Grande, v.7, p. 9-15, 2012. Disponível em: < http://www.seer.furg.br/revbea/article/view/1798/1682> Acesso em 04 Out. 2013.

DEMAJOROVIC J. VILELA AJ (Org.). Modelos e ferramentas de gestão ambiental: desafios e perspectivas para as organizações. SENAC: São Paulo, 2006.

ENGELMAN R; GUISSO RM; FRACASSO EM. Ações de Gestão Ambiental nas Instituições de Ensino Superior: O que tem sido feito. Revista de Gestão Social e Ambiental, v.3, n.1, p. 22-23, 2009. Disponível em: < http://www.revistargsa.org/rgsa/article/view/115/56 > Acesso em: 12 Mai 2013.

FERES YN; ANTUNES FZ. Gestão Ambiental em Instituições de Ensino: programa Ecoeficiência e Sistema de Gestão Ambiental do SENAC São Paulo. IX ENGEMA - Encontro Nacional sobre gestão empresarial e meio ambiente, 2007. Disponível em: <.http://www.casoe.com.br/wp-content/uploads/2012/11/f-GESTAO-AMBIENTAL-SENAC-2004.pdf> Acesso em: 03 Mai 2013.

GILONI-LIMA PC; LIMA VA; Gestão integrada de resíduos químicos em instituições de ensino superior. Revista Química Nova, v. 31, no 6, p. 1595 - 1598, 2008. Disponível em: < http://www.scielo.br/pdf/qn/ v31n6/a53v31n6.pdf > Acesso em: 12 Jun 2013. 
JACOBI, P \& BENSEN GR; Gestão de resíduos sólidos em São Paulo: Desafios da sustentabilidade. Estudos Avançados, v. 25, no 71, 2011. Disponível em < http://www.scielo.br/pdf/ea/v25n71/10.pdf >. Acesso em: 02 Set 2013.

MACEDO RG; PIMENTA HCD. Diagnóstico do gerenciamento de resíduos sólidos de uma instituição de ensino federal do Rio Grande do Norte. Revista de Engenharia Ambiental- Espírito Santo do Pinhal, v. 7, no 4, p. 211-231, out/dez.2010. Disponível em: <http://ferramentas.unipinhal.edu.br/ojs/engenhariaambiental/ include $/$ getdoc. php?id=1350\&article=568\&mode $=$ pdf. $>$ Acesso em: 02 Set 2013.

MONCKS RB; LIMA AS; PAZ MF; MARQUES RV; CORREA LB; CORRÊA EK; Gestão dos resíduos nos laboratórios de um instituição de ensino agronômico. In: 21 Congresso de Iniciação Científica \& 4 mostra Científica Universidade Federal de Pelotas. 2011. Disponível em: < http://www.ufpel.edu.br/cic/2012/ anais/pdf/EN/EN 00173.pdf> Acesso em: 20 Set 2013.

PEREIRA PS; OLIVEIRA LRA; SOUZA JGP; LEHFELD NAS; Resíduos Químicos: Gerenciamento em instituição de ensino e pesquisa. In: Congresso Interamericano de Ingeniería Sanitária y Ambiental. Punta Del Este, 26-30.2006. Disponível em: < http://www.bvsde.paho.org/bvsaidis/uruguay30/BR10551 Pereira. pdf > Acesso em: 05 Mai 2013.

REIS CC; Moraes JAR; Proposta de elaboração de política ambiental para Instituição de Ensino superior IES do Sul do País. In: $25^{\circ}$ congresso brasileiro de Engenharia sanitaria e ambiental; 2009. Rio Grande do Sul. Disponível em: < http://www.ufrgs.br/sga/SGA/material-de-apoio/textos/textos-apoio/links/VI-277.pdf>

Rocha CMC; Júnior AMM; Magalhães KM. Gestão de resíduos sólidos: Percepção ambiental de universitários em uma instituição de ensino superior brasileira. Revista Eletrônica de Mestrado em Educação Ambiental, v. 29, p. 1 - 12 julho a dezembro de 2012. Disponível em: < http://www.seer.furg.br/remea/article/ view/2962/1905> Acesso em: 10 Mai 2013.

Sartor MJ; Política de gerenciamento de resíduos nas universidades estaduais públicas Paraenses. Universidade Estadual de Londrina. Paraná. 2010. < http://www.uel.br/pos/economia/arq/DISSERTACOES/Sartor\%20Maria\%20Jose.pdf > Acesso em: 02 Jun. 2013.

Silva JRSS; Coelho BENC; Silva JP. Plano de gerenciamento de Resíduos Sólidos de uma Instituição de Ensino Superior do Estado de Goiás. In: III Congresso Brasileiro de Gestão Ambiental Goiânia, 19 a 22/11/2012. Disponível em: < http://www.ibeas.org.br/congresso/Trabalhos2012/I-013.pdf > Acesso em: 21 Junh 2013.

TAUCHEN J; BRANDLI LL; A gestão ambiental em instituições de ensino superior: modelo para implantação em campus universitário. Revista Gestão \& Produção. v.13, p. 503 - 515, 2006. Disponível em: < http:// www.scielo.br/pdf/gp/v13n3/11.pdf >Acesso em: 05 Abr 2013.

Termignoni, LDF. Framework de sustentabilidade para instituições de ensino superior comunitárias. Pontifícia Universidade Católica do Rio Grande do Sul. Porto Alegre. 2012. Disponível em: < http://www.ufrgs.br/ sga/SGA/material-de-apoio/textos/textos-apoio/links/Pagina\%20-\%20Material\%20de $\% 20$ Apoio\%20-\%20 Outros.pdf > Acesso em: 05 Mai 2013.

VAZ CR; FAGUNDES AB; KACHBA YR; OLIVEIRA IL; KOVALESKI JL; Sistema de Gestão Ambiental em Instituições de Ensino Superior: uma revisão. Revista GEPROS. Gestão da Produção, operações e sistemas, v.3, p. 45-58, jul/set. 2010. Ano 5. Disponível em: < http://revista.feb.unesp.br/index.php/gepros/article/viewFile/327/314 > Acesso em: 05 Mai 2013. 